\title{
Quantitative Autoradiographic Measurement of Local Cerebral Glucose Utilization in Freely Moving Rats During Postnatal Development
}

\author{
Astrid Nehlig, Anne Pereira de Vasconcelos, and Sylvette Boyet \\ INSERM U.272, 54013 Nancy Cedex, France
}

The quantitative $2-{ }^{14} \mathrm{C}$-deoxyglucose autoradiographic method of Sokoloff et al. (1977) was used to measure local cerebral glucose utilization in freely moving developing rats. The animals were studied at 10, 14, 17, 21, and $35 \mathrm{~d}$ and at the adult stage. Glucose utilization was very low and quite uniform in 10-and 14-d-old rats, ranging from 20 to $30 \mu \mathrm{mol} /$ $100 \mathrm{gm} / \mathrm{min}$, except in a few posterior areas. Between these 2 stages, rates of glucose utilization significantly increased in 6 areas, among which 4 were belonging to the auditory system. Between 14 and $17 \mathrm{~d}$, glucose utilization significantly changed in 9 structures out of the 68 studied, mainly auditory, visual, parietal, and thalamic areas. Between the stages of 17 and $21 \mathrm{~d}$, glucose utilization was increased by 50 or $100 \%$ in all brain structures studied, except in the medial habenula and white matter areas. After weaning time, rates of glucose utilization still significantly changed in $\mathbf{5 0}$ areas, widely distributed through all studied systems. Between $35 \mathrm{~d}$ and the adult stage, the average rate of glucose utilization did not change and rates of energy metabolism significantly increased in 13 brain areas. In one structure, the medial habenula, glucose utilization was already high 10 d after birth and did not change over the whole studied period. These increases in the rates of glucose utilization are consistent with the behavioral, anatomical, and functional changes known to occur during this period of development in the rat.

The development of the quantitative $2-{ }^{14} \mathrm{C}$-deoxyglucose autoradiographic method of Sokoloff et al. (1977) has made it possible to measure the rates of glucose utilization in each individual brain structure of conscious animals. This method has been extensively used to determine glucose utilization in discrete areas of the brain of adult animals in various physiological, pharmacological, and behavioral states (Sokoloff, 1981). Local cerebral metabolic rates for glucose (LCMRglu) have been shown to vary over a wide range and to be in a characteristic distri-

\footnotetext{
Received June 8, 1987; revised Nov. 4, 1987; accepted Nov. 6, 1987.

We wish to express our gratitude to Prof. Paul Vert, Director of INSFRM UI.272 for his encouragement, advice, and support. A. Nehlig is very grateful to Dr. J. Seylaz (Laboratoire de physiologie et physiopathologie cérébrovasculaire, Paris) for allowing her free access to the image-processing system facilities of his laboratory. This work was supported by the Institut National de la Santé et de la Recherche Médicale (U.272) and by a grant from the Fondation pour la Recherche Médicale (Comite Lorraine) covering the travel expenses for reading the autoradiograms. The technical assistance of $\mathrm{T}$. Colas and the editorial assistance of $\mathrm{E}$. Roussel are gratefully acknowledged.

Correspondence should be addressed to Dr. Astrid Nehlig, INSERM U.272, 30 rue Lionnois, B.P. 3137, 54013 Nancy Cedex, France.

Copyright $@ 1988$ Society for Neuroscience $0270-6474 / 88 / 072321-13 \$ 02.00 / 0$
}

bution in the brain of awake, conscious, mature animals (Sokoloff, 1981).

In vitro measurements of the rate of oxygen consumption and glucose utilization in the brain as a whole have shown that the cerebral metabolic rate is low at birth and increases with maturity (Fazekas et al., 1941; Himwich and Fazekas, 1941; Garfunkel et al., 1954; Duffy et al., 1975; Gregoire et al., 1978; Hernandez et al., 1978). Applications of the $2-{ }^{14} \mathrm{C}$-deoxyglucose autoradiographic method to newborn animals have demonstrated that LCMRglu are also heterogeneous throughout the brain of immature animals, but the values are lower and distributed over a smaller range than those obtained in the mature rat or monkey brain (Gregoire et al., 1981; Duffy et al., 1982; Kennedy et al., 1982; Abrams et al., 1984). In addition, the heterogeneity in the distribution of LCMRglu throughout the immature brain also varies as a function of the degrec of maturity of the different animal species at birth: dog (Duffy et al., 1975; Gregoire et al., 1981), monkey (Kato et al., 1980; Kennedy et al., 1982), or sheep (Abrams et al., 1984).

In 2 studies, the postnatal evolution of cerebral metabolic activity was followed during development. In the first, because of the close relationship between local cerebral blood flow and local cerebral metabolic and functional activity (Sokoloff, 1981), measurements of local cerebral blood flow by quantitative autoradiography permitted indirect assessment of local cerebral functional activity during development. In this study, Kennedy et al. (1972) could show that the rates of local cerebral blood flow in the dog measured with $\left[{ }^{14} \mathrm{C}\right]$ antipyrine were low and fairly uniform in the immediate postnatal period. The levels of blood flow exhibited a progressively increasing heterogeneity during the first weeks of life. The authors suggested that these changes in local cerebral blood flow reflected developmental differences in functional activity and energy metabolism of the brain. Recently, Chugani and Phelps (1986) and Chugani et al. (1987) measured the evolution of LCMRglu in human infants from age 5 weeks to 18 months with $\left[{ }^{18} \mathrm{~F}\right]$ fluorodeoxyglucose positron emission tomography. They were able to show that the pattern of cerebral functional activity changes is in good agreement with behavioral, neurophysiological, and anatomical alterations known to occur during infant development. However, no data is available on the postnatal evolution of LCMRglu in developing animals. Indeed, in previous studies, LCMRglu in newborn animals was only compared to a single other stage, to pubescent animals in monkeys (Kato et al., 1980; Kennedy et al., 1982) or to fetuses in the sheep (Abrams et al., 1984).

The $2-{ }^{14} \mathrm{C}$-deoxyglucose autoradiographic method needs for quantification to catheterize blood vessels and to collect timed 
arterial blood samples. For these reasons, $2-{ }^{14} \mathrm{C}$-deoxyglucose studies on immature animals have only been performed on those animal species with large youngs such as monkeys (Kato et al., 1980; Kennedy et al., 1982), beagle dogs (Duffy et al., 1982), and sheep (Abrams et al., 1984).

The present study represents the first attempt to apply the $2-{ }^{14} \mathrm{C}$-deoxyglucose method described by Sokoloff et al. (1977) to the quantitative autoradiographic measurement of the postnatal evolution of LCMRglu in freely moving rats between the age of $10 \mathrm{~d}$ after birth and the adult stage. The purpose of this study was to determine if there are changes in LCMRglu correlating to the functional, behavioral, and anatomical changes known to occur during this period of maturation. The rat was chosen in the present study because it is an easily available, currently used laboratory animal species. Its development after birth is rapid, achieving essentially adult behavior within 3-4 weeks after birth. In addition, the biochemical and anatomical correlates of the rat's cerebral development have been studied extensively.

\section{Materials and Methods}

Animals. Adult Spraguc-Dawley rats, 1 male and 2 females, were housed together in mating groups for $5 \mathrm{~d}$ and constantly maintained under standard laboratory conditions on a 12:12 hr light/dark cycle (lights on at $0600 \mathrm{hr}$ ). Food and water were available ad libitum. After delivery, litter sizes were reduced to 10 pups for homogeneity. Rat pups were weaned at $21 \mathrm{~d}$. The experiments were performed on a total of 36 animals at $10,14,17,21$, and $35 \mathrm{~d}$ after birth and at the adult stage. Before weaning, both male and female rat pups were used, but only male animals were used after weaning.

A femoral artery and vein were catheterized with polyethylene tubing under light halothane anesthesia. Both catheters were threaded under the skin, up to the neck through a small opening in the skin. A loop was made with the ends of each catheter, which were put back under the skin, and the small opening in the skin was closed. The animals were returned to their usual environment, back to their mother for suckling animals and allowed food and water ad libitum. The experiments were performed on the following day. Only those animals that did not suffer from the surgery and did not lose weight (especially for suckling rats, which were sometimes rejected by the mother) were used for the study.

Measurement of local cerebral glucose utilization. On the day of the experiment, the rats were transferred to an uncovered plastic box of variable size according to the studied stage, allowing easy access to catheters as well as free movement of the animals. The threads on the skin in the neck were cut and the catheters were pulled out.

I C.MRglu were measured by the $2-{ }^{14} \mathrm{C}$-deoxyglucose method described by Sokoloff et al. (1977). The $2-{ }^{14} \mathrm{C}$-deoxyglucose $(125 \mu \mathrm{Ci} / \mathrm{kg}$; specific activity, $50-55 \mathrm{mCi} / \mathrm{mmol}$; New England Nuclear, Boston) was injected as an intravenous pulse. Nine to 20 timed arterial blood samples were drawn during the following $45 \mathrm{~min}$ in glass capillary tubes for developing animals and in small plastic tubes for adults. The volumes of the samples ranged from 10 to $75 \mu \mathrm{l}$ according to the animal's age. Arterial blood samples were immediately centrifuged in a Hettich Microrapid microfuge, and the plasma concentration of $2-{ }^{14} \mathrm{C}$-deoxyglucose was determined by liquid scintillation counting on 3-20 $\mu \mathrm{l}$ samples in a Beckman scintillation counter (model LS 1801, Beckman Instruments, Inc. Fullerton, CA). Plasma glucose concentrations were assessed from $10 \mu \mathrm{l}$ of plasma by means of a Beckman glucose analyzer, on 3 larger samples from small animals and on all samples from adults.

Approximately $45 \mathrm{~min}$ after the pulse of $2-{ }^{14} \mathrm{C}$-deoxyglucose, the animals were killed by decapitation. Brains were rapidly removed and frozen in isopentane chilled to $-40^{\circ} \mathrm{C}$, coated with chilled embedding medium (M-1 Embedding Matrix, Lipshaw Manufacturing Co., Detroit), and stored at $-80^{\circ} \mathrm{C}$ in plastic bags until sectioned and autoradiographed. The brains were then cut into $20 \mu \mathrm{m}$ coronal sections at $-22^{\circ} \mathrm{C}$ in a cryostat. Sections were picked up on glass coverslips and dried on a hot plate $\left(60^{\circ} \mathrm{C}\right)$. Sections were autoradiographed on Kodak SB5 film along with the calibrated ${ }^{14} \mathrm{C}$-methylmethacrylate standards (Amersham, Arlington Heights, IL), using a special set of low- ${ }^{14} \mathrm{C}$ concentration standards for animals 10,14 , and $17 \mathrm{~d}$ old. All standards were calibrated for their ${ }^{14} \mathrm{C}$ concentration in brain sections, as previously described (Sokoloff et al., 1977). Adjacent sections were fixed and stained with thionin for histological identification of specific nuclei.

The autoradiographs were analyzed by quantitative densitometry with a computerized image-processing system (Histopericolor, Numelec, Biocom, France) or a manual microdensitometer (Macbeth, TD 901, Kollmorgen Co., Newburgh, NY). Optical density measurements for each structure anatomically defined according to the rat brain atlas of Paxinos and Watson (1982) for adult animals and to the developing rat brain atlas of Sherwood and Timiras (1970) for all other stages were made bilaterally in a minimum of 4 brain sections. Tissue ${ }^{14} \mathrm{C}$ concentrations were determined from the optical densities of the autoradiographic representations of the tissucs and a calibration curve obtained from the autoradiographs of the calibrated standards.

Calculation of local cerebral glucose utilization. The lumped constant and rate constants used for the calculation of LCMRglu in the operational equation of the method have been determined in the adult rat (Sokoloff et al., 1977). Because of the small size of the developing animals, it was impossible to measure the lumped and rate constants in pups. Instead, the values of the constants obtained in adult rats were used for calculations at all developmental stages. Indeed, the lumped constant was compared at 2 developmental stages in a single study on fetal and neonatal sheep and was found to be the same at both stages (Abrams et al., 1984). In studies on the monkey, the lumped constant measured in the adult brain was used indifferently for both newborn, pubescent, and adult animals (Kennedy et al., 1978, 1982; Kato et al., 1980). For the rate constants, even if the values determined in the adult rats do not apply exactly to rat pups, the magnitude of the error introduced by the use of approximate values is minimized by the fact that all the terms in the equation that contain the rate constants approach zero as the time between the isotope injection and killing increases. Indeed, after $45 \mathrm{~min}$ experimental period, these terms reach very small values, never exceeding $4-6 \%$ of the final plasma specific integrated activity for $2-{ }^{14} \mathrm{C}$-deoxyglucose calculated in the present study.

Also, for the final calculations, the exact time of blood sampling was corrected for the time lag due to the residual volume of blood in the catheter. The lag was calculated from the volume of the arterial catheter and the blood flow value. It ranged from 2 to $6 \mathrm{sec}$ and was subtracted to the recorded time of sampling.

LCMRglu was then calculated from the local tissue concentration of ${ }^{14} \mathrm{C}$, the time courses of the plasma $2-{ }^{14} \mathrm{C}$-deoxyglucose and glucose concentrations, and the appropriate contants according to the operational equation of the method (Sokoloff et al., 1977).

Measurement of blood $\beta$-hydroxybutyrate and acetoacetate levels. Total blood was collected into a preheparinized glass dish after decapitation of a separate group of rats, deproteinized by chilled $10 \%(\mathrm{vol} / \mathrm{vol}) \mathrm{per}-$ chloric acid, and centrifuged at $3000 \times g$ for $15 \mathrm{~min}$ at $2^{\circ} \mathrm{C}$. The supernatant solution, previously neutralized by $20 \% \mathrm{KOH}$ was used for enzymatic and spectrophotometric determination of $\beta$-hydroxybutyrate (Williamson and Mellanby, 1974) and acetoacetate (Mellanby and Williamson, 1974) by means of a Bausch and Lomb spectrophotometer (model Spectronic 2000, Bausch and Lomb, Analytical Systems Division, Rochester, NY).

Physiological variables. Just prior to the administration of the $2-{ }^{14} \mathrm{C}$ deoxyglucose pulse, mean arterial blood pressure of the animals was measured with an air-damped mercury manometer. Suckling rat pups were maintained under a heating lamp to keep their body temperature in the normal range. The hematocrit value was determined at about 30 min after the injection of the radioactive tracer. Arterial $\mathrm{pH}, \mathrm{pO}_{2}$ and $\mathrm{pCO}_{2}$ were measured on 40 or $80 \mu \mathrm{l}$ blood samples by means of a blood gas analyzer (Corning, model 158, Le Vesinet, France) just before sacrifice of the animals.

Statistical analysis. LCMRglu was determined in 68 cerebral structures in 6 groups of 6 animals. LCMRglu in each group of animals was compared with those in the immediate preceding stage by means of Bonferoni multiple comparison procedures (Kirk, 1968). Conservative multiple comparison procedures were chosen to reduce the likelihood of type II errors in view of the large number of statistical procedures performed.

\section{Results}

\section{Physiological variables}

Whereas the body weight of the rats increased by $100 \%$ between day 10 and 21 after birth, body growth was twice as fast between 
Table 1. Physiolngical variables in rats at different developmental stages

\begin{tabular}{|c|c|c|c|c|c|c|}
\hline Variable & $\operatorname{P10}(n=6)$ & $\mathrm{P} 14(n=6)$ & $\mathrm{P} 17(n=6)$ & $\mathrm{P} 21(n=6)$ & $\mathrm{P} 35(n=6)$ & Adult $(n=6)$ \\
\hline Body weight (gm) & $23 \pm 1$ & $33 \pm 3$ & $42 \pm 2$ & $48 \pm 1$ & $141 \pm 5^{c}$ & $312 \pm 5$ \\
\hline Plasma glucose concentration $(\mathrm{mg} / 100 \mathrm{ml})$ & $127 \pm 2$ & $137 \pm 5$ & $146 \pm 3$ & $131 \pm 3$ & $155 \pm 8^{b}$ & $166 \pm 10$ \\
\hline Blood $\beta$-hydroxybutyrate concentration ${ }^{a}(\mu \mathrm{mol} / \mathrm{ml})$ & $1.04 \pm 0.10$ & $1.09 \pm 0.10$ & - & $0.29 \pm 0.04$ & $0.15 \pm 0.01^{c}$ & - \\
\hline Blood acetoacetate concentration ${ }^{a}(\mu \mathrm{mol} / \mathrm{ml})$ & $0.33 \pm 0.02$ & $0.31 \pm 0.01$ & - & $0.13 \pm 0.02^{c}$ & $0.10 \pm 0.01$ & - \\
\hline Arterial blood pressure $(\mathrm{mm} \mathrm{Hg})$ & $51 \pm 2$ & $54 \pm 2$ & $66 \pm 2^{c}$ & $80 \pm 1$ & $102 \pm 2$ & $116 \pm 2$ \\
\hline Hematocrit (\%) & $32 \pm 1$ & $32 \pm 1$ & $32 \pm 1$ & $36 \pm 1$ & $45 \pm 1^{c}$ & $47 \pm 1$ \\
\hline $\mathrm{pH}$ & $7.48 \pm 0.02$ & $7.43 \pm 0.02$ & $7.45 \pm 0.01$ & $7.47 \pm 0.01$ & $7.45 \pm 0.01$ & $7.44 \pm 0.02$ \\
\hline Arterial $\mathrm{pO}_{2}(\mathrm{~mm} \mathrm{Hg})$ & $85 \pm 1$ & $86 \pm 2$ & $88 \pm 1$ & $87 \pm 1$ & $85 \pm 2$ & $84 \pm 2$ \\
\hline Arterial $\mathrm{pCO}_{2}(\mathrm{~mm} \mathrm{Hg})$ & $28 \pm 1$ & $35 \pm 1^{c}$ & $33 \pm 1$ & $32 \pm 2$ & $35 \pm 1$ & $35 \pm 1$ \\
\hline
\end{tabular}

Values are means \pm SEM of the number of animals given in parentheses.

" From Pereira de Vasconcelos et al. (1987).

${ }^{n} p<0.05$.

$c p<0.01$ (statistically significant difference for each developmental stage).

21 and $35 \mathrm{~d}$ of age as during the preceding period (Table 1). Plasma glucose concentration and the hematocrit value did not change from day 10 to 21 after birth but increased significantly between 21 and $35 \mathrm{~d}$. Blood levels of $\beta$-hydroxybutyrate and acetoacetate were high at the 2 first stages studied and were decreased by 3-4 times at weaning.

Arterial $\mathrm{pH}, \mathrm{pO}_{2}$, and $\mathrm{pCO}_{2}$ did not change over the whole period studied, except for $\mathrm{pCO}_{2}$, which increased between days 10 and 14 after birth.

\section{Variations in local cerebral glucose utilization with postnatal} development

LCMRglu increased over the whole postnatal period studied (Tables 2-6), especially by $21 \mathrm{~d}$ after birth. At the first 3 stages studied (i.e., P10, P14 and P17), LCMRglu exhibited uniformly low values throughout the brain.

\section{P10 animals}

LCMRglu was generally very low, ranging from 20 to $30 \mu \mathrm{mol} /$ $100 \mathrm{gm} / \mathrm{min}$ at $10 \mathrm{~d}$ after birth (Tables 2-6, Figs. 1-4). Only a few structures exhibited higher values. These were mostly quite posterior areas, such as the raphe nuclei, the dorsal tegmental nucleus (Table 3), the inferior olive (Table 5), and vestibular and cochlear nuclei (Table 2, Fig. 4). A few more anterior areas also had higher rates of glucose utilization: these were the medial habenula (Fig. 2) and interpeduncular nucleus (Table 3), the paraventricular nucleus (Table 4, Fig. 1), and substantia nigra pars compacta (Table 5), which appeared as early as $10 \mathrm{~d}$ after birth as a small dark line (Fig. 3).

\section{P14 animals}

At $14 \mathrm{~d}$ after birth, LCMRglu was still quite low and uniform throughout the brain (Tables 2-6, Figs. 1-4). It did not change

Table 2. Local cerebral glucose utilization in sensory systems of rats at different developmental stages

\begin{tabular}{|c|c|c|c|c|c|c|}
\hline System & $\mathrm{P} 10(n=6)$ & $\mathrm{P} 14(n=6)$ & $\mathrm{P} 17(n=6)$ & $\mathrm{P} 21(n=6)$ & $\mathrm{P} 35(n=6)$ & Adult $(n=6)$ \\
\hline \multicolumn{7}{|l|}{ Visual } \\
\hline Visual cortex & $20 \pm 2$ & $24 \pm 1$ & $32 \pm 3$ & $63 \pm 3^{b}$ & $87 \pm 5^{b}$ & $97 \pm 5$ \\
\hline Lateral geniculate body & $20 \pm 2$ & $27 \pm 2$ & $37 \pm 2^{a}$ & $53 \pm 2^{b}$ & $76 \pm 3^{b}$ & $75 \pm 3$ \\
\hline Superior colliculus & $20 \pm 1$ & $26 \pm 1$ & $32 \pm 3$ & $52 \pm 1^{b}$ & $63 \pm 2^{b}$ & $76 \pm 3^{\prime \prime}$ \\
\hline \multicolumn{7}{|l|}{ Auditory } \\
\hline Auditory cortex & $20 \pm 1$ & $30 \pm 2$ & $50 \pm 4^{b}$ & $86 \pm 6^{b}$ & $112 \pm 5^{b}$ & $145 \pm 4^{h}$ \\
\hline Medial geniculate body & $23 \pm 1$ & $36 \pm 3^{a}$ & $49 \pm 3^{a}$ & $69 \pm 3^{b}$ & $98 \pm 3^{b}$ & $114 \pm 4^{h}$ \\
\hline Inferior colliculus & $22 \pm 2$ & $53 \pm 8^{a}$ & $62 \pm 7$ & $101 \pm 5^{b}$ & $136 \pm 8^{b}$ & $164 \pm 5^{b}$ \\
\hline Lateral lemniscus & $26 \pm 2$ & $47 \pm 4^{a}$ & $53 \pm 4$ & $79 \pm 4^{b}$ & $83 \pm 7$ & $89 \pm 5$ \\
\hline Superior olive & $31 \pm 1$ & $62 \pm 7^{b}$ & $66 \pm 4$ & $90 \pm 5^{b}$ & $101 \pm 5$ & $117 \pm 4$ \\
\hline Cochlear nucleus & $48 \pm 1$ & $46 \pm 1$ & $54 \pm 3$ & $73 \pm 2^{b}$ & $92 \pm 6^{b}$ & $100 \pm 4$ \\
\hline \multicolumn{7}{|l|}{ Olfactory } \\
\hline Olfactory cortex & $29 \pm 3$ & $36 \pm 2$ & $39 \pm 2$ & $60 \pm 3^{b}$ & $85 \pm 3^{b}$ & $78 \pm 4$ \\
\hline \multicolumn{7}{|l|}{ Somatic } \\
\hline \multicolumn{7}{|l|}{ Frontoparietal cortex, } \\
\hline Facial nerve nucleus & $37 \pm 2$ & $37 \pm 2$ & $38 \pm 2$ & $54 \pm 2^{b}$ & $63 \pm 4^{a}$ & $57 \pm 1$ \\
\hline Spinal trigeminnal nucleus & $27 \pm 2$ & $33 \pm 2$ & $35 \pm 2$ & $46 \pm 1^{b}$ & $58 \pm 3^{b}$ & $56 \pm 1$ \\
\hline \multicolumn{7}{|l|}{ Vestibular } \\
\hline Vestibular nucleus & $49 \pm 2$ & $53 \pm 3$ & $56 \pm 2$ & $71 \pm 3^{b}$ & $93 \pm 4^{b}$ & $101 \pm 4$ \\
\hline
\end{tabular}

Values are means \pm SEM of the number of animals given in parentheses, expressed as $\mu \mathrm{mol} / 100 \mathrm{gm} / \mathrm{min}$.

" $p<0.01$ (statistically significant difference for each developmental stage). 
Table 3. Local cerebral glucose utilization in limbic and functionally nonspecific areas of rats at different developmental stages

\begin{tabular}{|c|c|c|c|c|c|c|}
\hline Structure & $\mathrm{P} 10(n=6)$ & $\mathrm{P} 14(n=6)$ & $\mathrm{P} 17(n=6)$ & $\mathrm{P} 21(n=6)$ & $\mathrm{P} 35(n=6)$ & Adult $(n=6)$ \\
\hline Prefrontal cortex & $22 \pm 3$ & $30 \pm 2$ & $37 \pm 2$ & $65 \pm 3^{b}$ & $93 \pm 2^{b}$ & $103 \pm 5$ \\
\hline Anterior cingulate cortex & $22 \pm 3$ & $28 \pm 1$ & $32 \pm 2$ & $62 \pm 3^{b}$ & $83 \pm 3^{h}$ & $98 \pm 4^{a}$ \\
\hline Entorhinal cortex & $21 \pm 2$ & $24 \pm 2$ & $29 \pm 2$ & $42 \pm 2^{b}$ & $54 \pm 4^{b}$ & $58 \pm 2$ \\
\hline Nucleus accumbens & $25 \pm 3$ & $28 \pm 1$ & $33 \pm 2$ & $54 \pm 4^{b}$ & $65 \pm 2^{a}$ & $74 \pm 1^{a}$ \\
\hline Medial septum & $26 \pm 1$ & $36 \pm 2$ & $35 \pm 1$ & $52 \pm 4^{h}$ & $70 \pm 4^{b}$ & $75 \pm 3$ \\
\hline Lateral septum & $23 \pm 1$ & $26 \pm 1$ & $26 \pm 1$ & $37 \pm 3^{b}$ & $43 \pm 2$ & $46 \pm 1$ \\
\hline Medial amygdala & $22 \pm 2$ & $24 \pm 2$ & $28 \pm 2$ & $39 \pm 2^{b}$ & $41 \pm 2$ & $46 \pm 2$ \\
\hline Central amygdala & $22 \pm 2$ & $22 \pm 1$ & $27 \pm 2$ & $35 \pm 1^{a}$ & $37 \pm 2$ & $41 \pm 2$ \\
\hline Basolateral amygdala & $24 \pm 3$ & $28 \pm 2$ & $35 \pm 2$ & $52 \pm 3^{b}$ & $63 \pm 3^{a}$ & $68 \pm 2$ \\
\hline Dorsal hippocampus & $30 \pm 2$ & $28 \pm 2$ & $33 \pm 2$ & $47 \pm 2^{b}$ & $59 \pm 2^{b}$ & $63 \pm 3$ \\
\hline Ventral hippocampus & $28 \pm 2$ & $30 \pm 2$ & $32 \pm 2$ & $45 \pm 2^{b}$ & $58 \pm 3$ & $64 \pm 2^{h}$ \\
\hline Dentate gyrus & - & $23 \pm 2$ & $26 \pm 1$ & $33 \pm 1^{b}$ & $39 \pm 2$ & $46 \pm 3^{a}$ \\
\hline Mediodorsal thalamus & $27 \pm 3$ & $30 \pm 2$ & $40 \pm 3^{a}$ & $65 \pm 3^{h}$ & $81 \pm 3^{\prime \prime}$ & $95 \pm 2^{b}$ \\
\hline Medial habenula & $56 \pm 2$ & $60 \pm 2$ & $56 \pm 1$ & $6 C \pm 3$ & $60 \pm 3$ & $54 \pm 1$ \\
\hline Lateral habenula & $36 \pm 1$ & $44 \pm 2^{a}$ & $51 \pm 1$ & $71 \pm 2^{b}$ & $88 \pm 3^{h}$ & $86 \pm 2$ \\
\hline Interpeduncular nucleus & $42 \pm 3$ & $46+2$ & $51+3$ & $72 \pm 3^{b}$ & $77+1$ & $83 \pm 4$ \\
\hline Ventral tegmental area & $28 \pm 1$ & $35 \pm 2$ & $41 \pm 2$ & $53 \pm 3^{b}$ & $60 \pm 4$ & $57 \pm 1$ \\
\hline Dorsal tegmental nucleus & $50 \pm 2$ & $42 \pm 2$ & $44 \pm 3$ & $57 \pm 4^{a}$ & $67 \pm 4$ & $86 \pm 3^{n}$ \\
\hline Mesencephalic reticular formation & $24 \pm 1$ & $30 \pm 2$ & $36 \pm 2$ & $50 \pm 3^{b}$ & $62 \pm 3^{b}$ & $62 \pm 1$ \\
\hline Medial raphe & $42 \pm 1$ & $42 \pm 2$ & $42 \pm 1$ & $72 \pm 4^{b}$ & $85 \pm 5^{h}$ & $91 \pm 2$ \\
\hline Dorsal raphe & $36 \pm 2$ & $35 \pm 2$ & $39 \pm 1$ & $66 \pm 3^{b}$ & $82 \pm 3^{n}$ & $81 \pm 1$ \\
\hline Locus coeruleus & $32 \pm 1$ & $33 \pm 2$ & $32 \pm 2$ & $53 \pm 3^{b}$ & $65 \pm 4^{h}$ & $60 \pm 1$ \\
\hline Pontine gray & $24 \pm 2$ & $26 \pm 1$ & $30 \pm 2$ & $50 \pm 2^{b}$ & $56 \pm 5$ & $59 \pm 4$ \\
\hline
\end{tabular}

Values are means \pm SEM of the number of animals given in parentheses, expressed as $\mu \mathrm{mol} / 100 \mathrm{gm} / \mathrm{min}$.

$" p<0.05$.

${ }^{\prime} p<0.01$ (statistically significant difference for each developmental stage).

in most brain areas studied between 10 and $14 \mathrm{~d}$. The increases in LCMRglu were mainly located in primary auditory areas such as the medial geniculate body, inferior colliculus, lateral lemniscus, and superior olive (Table 2), as well as in the lateral habenula (Table 3) and subthalamic nucleus (Table 5).

\section{PI7 animals}

At $17 \mathrm{~d}$ after birth, LCMRglu slightly increased compared with the 2 preceding stages but remained quite low and uniform throughout the brain, ranging from 30 to $40 \mu \mathrm{mol} / 100 \mathrm{gm} / \mathrm{min}$. Between 14 and $17 \mathrm{~d}$ after birth, LCMRglu increased in 10 of
68 structures studied. These were the auditory cortex, medial geniculate (Fig. 3), and lateral geniculate bodies (Table 2), the 2 frontoparietal cortices, somatosensory and motor areas (Tables 2 and 5), the mamillary body (Table 4), red nucleus, inferior olive, ventroanterior (Table 4), and mediodorsal thalamic nuclei (Table 3).

\section{P21 animals}

At $21 \mathrm{~d}$ after birth, LCMRglu appeared to be more differentiated in most brain areas (Figs. 1-4). Between 17 and $21 \mathrm{~d}$ after birth, LCMRglu increased in almost all structures studied by $50-100 \%$

\begin{tabular}{|c|c|c|c|c|c|c|}
\hline Structure & $\mathrm{P} 10(n=6)$ & $\mathrm{P} 14(n=6)$ & $\mathrm{P} 17(n=6)$ & P21 $(n=6)$ & $\mathrm{P} 35(n=6)$ & Adult $(n=6)$ \\
\hline Medial preoptic area & $22 \pm 1$ & $22 \pm 1$ & $25 \pm 1$ & $33 \pm 2^{a}$ & $43 \pm 4^{a}$ & $42 \pm 2$ \\
\hline Lateral preoptic area & $33 \pm 1$ & $35 \pm 2$ & $37 \pm 2$ & $53 \pm 2^{b}$ & $73 \pm 5^{\prime \prime}$ & $70 \pm 3$ \\
\hline Magnocellular preoptic area & $34 \pm 1$ & $43 \pm 2$ & $44 \pm 2$ & $64 \pm 3^{\prime \prime}$ & $84 \pm 5^{\prime \prime}$ & $79 \pm 3$ \\
\hline Suprachiasmatic nucleus & $43 \pm 1$ & $40 \pm 3$ & $44 \pm 2$ & $51 \pm 3$ & $68 \pm 4^{\prime \prime}$ & $65 \pm 3$ \\
\hline Anterior hypothalamus & $21 \pm 1$ & $26 \perp 1$ & $28 \pm 2$ & $43 \pm 3^{b}$ & $53 \pm 3^{n}$ & $47 \pm 1$ \\
\hline Anterolateral hypothalamus & $27 \pm 1$ & $32 \pm 2$ & $33 \pm 2$ & $49 \pm 3^{b}$ & $61 \pm 3^{\prime \prime}$ & $54 \pm 1$ \\
\hline Paraventricular nucleus & $33 \pm 2$ & $32 \pm 2$ & $30 \pm 2$ & $43 \pm 3^{b}$ & $52 \pm 2^{a}$ & $46+2$ \\
\hline Ventromedial hypothalamus & $21 \pm 1$ & $23 \pm 2$ & $25 \pm 2$ & $38 \pm 2^{b}$ & $48 \pm 1^{\prime \prime}$ & $44 \pm 2$ \\
\hline Dorsomedial hypothalamus & $25 \pm 1$ & $27 \pm 1$ & $29 \pm 2$ & $47 \pm 2^{b}$ & $59 \pm 3^{\prime \prime}$ & $52 \pm 1$ \\
\hline Medial forebrain bundle & $29 \pm 2$ & $33 \pm 2$ & $36 \pm 2$ & $52 \pm 2^{b}$ & $63 \pm 3^{b}$ & $56 \pm 1$ \\
\hline Posterior hypothalamus & $19 \pm 1$ & $25 \pm 1$ & $29 \pm 2$ & $40 \pm 3^{b}$ & $46 \pm 2$ & $46 \pm 2$ \\
\hline Mamillary body & $28 \pm 1$ & $38 \pm 3$ & $57 \pm 3^{b}$ & $86 \pm 5^{h}$ & $101 \pm 6^{a}$ & $103 \pm 4$ \\
\hline
\end{tabular}

Values are means $\pm \mathrm{SEM}$ of the number of animals given in parentheses, expressed as $\mu \mathrm{mol} / 100 \mathrm{gm} / \mathrm{min}$.

" $p<0.05$.

" $p<0.01$ (statistically significant difference for each developmental stage). 


\begin{tabular}{|c|c|c|c|c|c|c|}
\hline Structure & $\mathrm{P} 10(n=6)$ & $\mathrm{P} 14(n=6)$ & $\mathrm{P} 17(n=6)$ & $\mathrm{P} 21(n=6)$ & P35 $(n=6)$ & Adult $(n=6)$ \\
\hline Frontoparietal cortex motor area & $25 \pm 2$ & $33 \pm 2$ & $43 \pm 2^{a}$ & $65 \pm 3^{a}$ & $79 \pm 2^{b}$ & $103 \pm 3^{\prime \prime}$ \\
\hline Dorsomedial caudate nucleus & $23 \pm 4$ & $29 \pm 1$ & $35 \pm 3$ & $59 \pm 3^{b}$ & $82 \pm 4^{b}$ & $88 \pm 4$ \\
\hline Dorsolateral caudate nucleus & $27 \pm 4$ & $30 \pm 2$ & $37 \pm 3$ & $53 \pm 2^{b}$ & $75 \pm 3^{b}$ & $82 \pm 4$ \\
\hline Ventral caudate nucleus & $25 \pm 3$ & $29 \pm 1$ & $35 \pm 3$ & $52 \pm 2^{b}$ & $72 \pm 4^{b}$ & $84 \pm 2^{\prime \prime}$ \\
\hline Globus pallidus & $23 \pm 3$ & $24 \pm 1$ & $29 \pm 2$ & $38 \pm 1^{\prime \prime}$ & $43 \pm 1$ & $46 \pm 2$ \\
\hline Substantia nigra, pars reticulata & $27 \pm 2$ & $31 \pm 2$ & $37 \pm 2$ & $47 \pm 2^{h}$ & $55 \pm 2^{b}$ & $52 \pm 1$ \\
\hline Substantia nigra, pars compacta & $35 \pm 2$ & $40 \pm 2$ & $41 \pm 2$ & $52 \pm 3^{h}$ & $66 \pm 3^{h}$ & $62 \pm 1$ \\
\hline Subthalamic nucleus & $27 \pm 1$ & $36 \pm 2^{\prime}$ & $41 \pm 1$ & $57 \pm 2^{b}$ & $75 \pm 3^{h}$ & $71 \pm 1$ \\
\hline Ventroanterior thalamus & $33 \pm 4$ & $32 \pm 2$ & $46 \pm 4^{b}$ & $64 \pm 2^{b}$ & $83 \pm 3^{l}$ & $90 \pm 1$ \\
\hline Ventrolateral thalamus & $26 \pm 2$ & $30 \pm 2$ & $38 \pm 3$ & $57 \pm 3^{h}$ & $75 \pm 3^{h}$ & $84 \pm 1^{a}$ \\
\hline Red nucleus & $28 \pm 1$ & $34 \pm 2$ & $43 \pm 2^{a}$ & $55 \pm 3^{h}$ & $62 \pm 3$ & $69 \pm 2$ \\
\hline Inferior olive & $46 \pm 2$ & $46 \pm 3$ & $49 \pm 2$ & $63 \pm 5^{b}$ & $76 \pm 4^{a}$ & $72 \pm 3$ \\
\hline Cerebellar cortex & $27 \pm 3$ & $20 \pm 2$ & $20 \pm 1$ & $33 \pm 1^{b}$ & $43 \pm 2^{h}$ & $44 \pm 2$ \\
\hline \multicolumn{7}{|l|}{ Cerebellar nuclei } \\
\hline Dentate nucleus & $34 \pm 2$ & $37 \pm 3$ & $40 \pm 1$ & $60 \pm 4^{h}$ & $77 \pm 4^{h}$ & $77 \pm 1$ \\
\hline Fastigial nucleus & $37 \pm 2$ & $39 \pm 2$ & $44 \pm 2$ & $59 \pm 3^{b}$ & $75 \pm 3^{\prime \prime}$ & $75 \pm 2$ \\
\hline Interpositus nucleus & $41 \pm 2$ & $39 \pm 2$ & $43 \pm 1$ & $57 \pm 2^{b}$ & $77 \pm 3^{\prime \prime}$ & $77 \pm 3$ \\
\hline
\end{tabular}

Values are means \pm SEM of the number of animals given in parentheses, expressed as $\mu \mathrm{mol} / 100 \mathrm{gm} / \mathrm{min}$.

$" p<0.05$.

" $p<0.01$ (statistically significant difference for each developmental stage).

(Tables 2-6), as reflected by the increase in the average rate of glucose utilization in the brain as a whole, weighted for the relative sizes of its component parts (Table 6). LCMRglu was unchanged in only 5 of the 68 brain structures studied. These were the medial habenula (Table 3), the suprachiasmatic nucleus (Table 4), and the three white matter areas: the genu, internal capsule, and cerebellar white matter (Table 6).

\section{P35 animals}

Between 21 and $35 \mathrm{~d}$, LCMRglu underwent a statistically significant increase in 50 of the 68 studied cerebral structures. The average rate of glucose utilization of the brain as a whole, weightcd for the relative sizes of component parts, increased by $25 \%$ between P21 and P35. Increases in LCMRglu occurred in all sensory areas except for 2 auditory structures, the lateral lemniscus and superior olive (Table 2). LCMRglu increased in 13 of the 23 limbic and functionally nonspecific structures studied (Table 3). Statistically significant increases in LCMRglu between P21 and P35 were also noted in all hypothalamic areas, except the posterior hypothalamus (Table 4) and in all extrapyramidal and sensorimotor areas, except in the globus pallidus and red nucleus (Table 5).

\section{Adult animals}

LCMRglu underwent statistically significant increases in 14 of the 68 structures studied between day 35 and adulthood. These changes were located mainly in primary visual and auditory areas (Table 2), in 6 limbic areas (Table 3) and in 3 extrapyramidal and sensorimotor structures: the frontoparietal cortex, motor area, the ventral caudate nucleus, and the ventrolateral thalamus (Table 5). There was no change in LCMRglu in any hypothalamic structure between P35 and adulthood (Table 4).

LCMRglu was unchanged throughout the whole developmental period studied in one gray matter area, the medial habenula (Table 4, Fig. 2) and in the 3 white matter areas (Table 6).

\section{Discussion}

The results of the present study represent, to our knowledge, the first quantitative measurement of LCMRglu in rats during postnatal development. These results show that LCMRglu is very low and quite homogeneous, except in a few posterior areas, at the earliest stages, i.e., at P10 and P14. LCMRglu increased 2- to 3 -fold in most of the brain structures studied and became

Table 6. Local cerebral glucose utilization in white matter of rats at different developmental stages

\begin{tabular}{lllllll} 
Structure & $\mathrm{P} 10(n=6)$ & $\mathrm{P} 14(n=6)$ & $\mathrm{P} 17(n=6)$ & $\mathrm{P} 21(n=6)$ & P35 $(n=6)$ & Adult $(n=6)$ \\
\hline Genu of the corpus callosum & $19 \pm 2$ & $20 \pm 1$ & $22 \pm 2$ & $26 \pm 1$ & $25 \pm 1$ \\
Internal capsule & $19 \pm 2$ & $22 \pm 1$ & $28 \pm 2$ & $34 \pm 1$ & $31 \pm 2$ & $32 \pm 3$ \\
Cerebellar white matter & - & - & - & $40 \pm 1$ & $41 \pm 3$ \\
Weighted average & $24 \pm 2$ & $29 \pm 2$ & $34 \pm 2$ & $51 \pm 3^{a}$ & $64 \pm 3^{a}$ & $67 \pm 2$
\end{tabular}

Values are means \pm SEM of the number of animals given in parentheses, expressed as $\mu \mathrm{mol} / 100 \mathrm{gm} / \mathrm{min}$.

" $p<0.01$ (statistically significant difference for each developmental stage). 

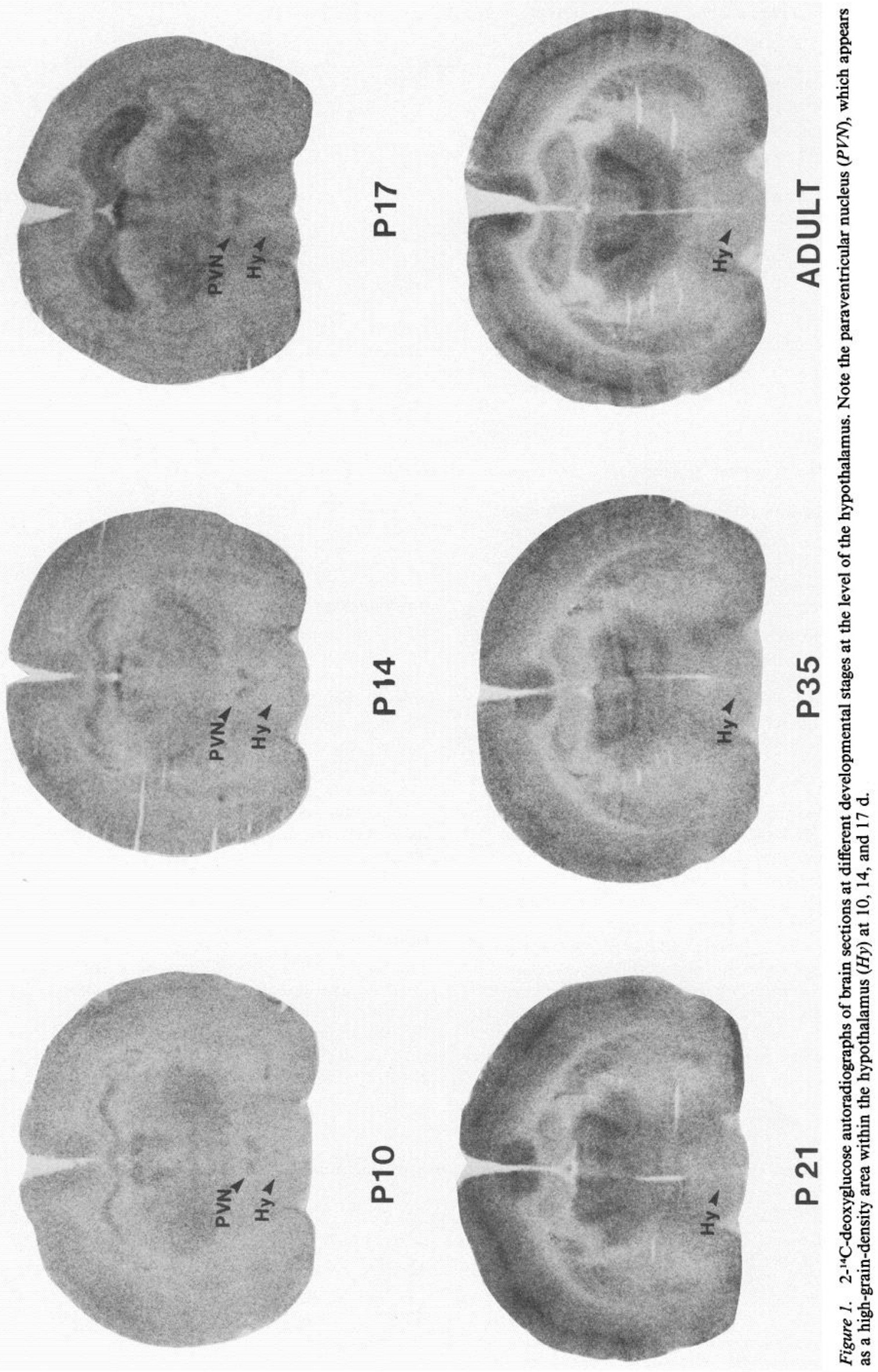

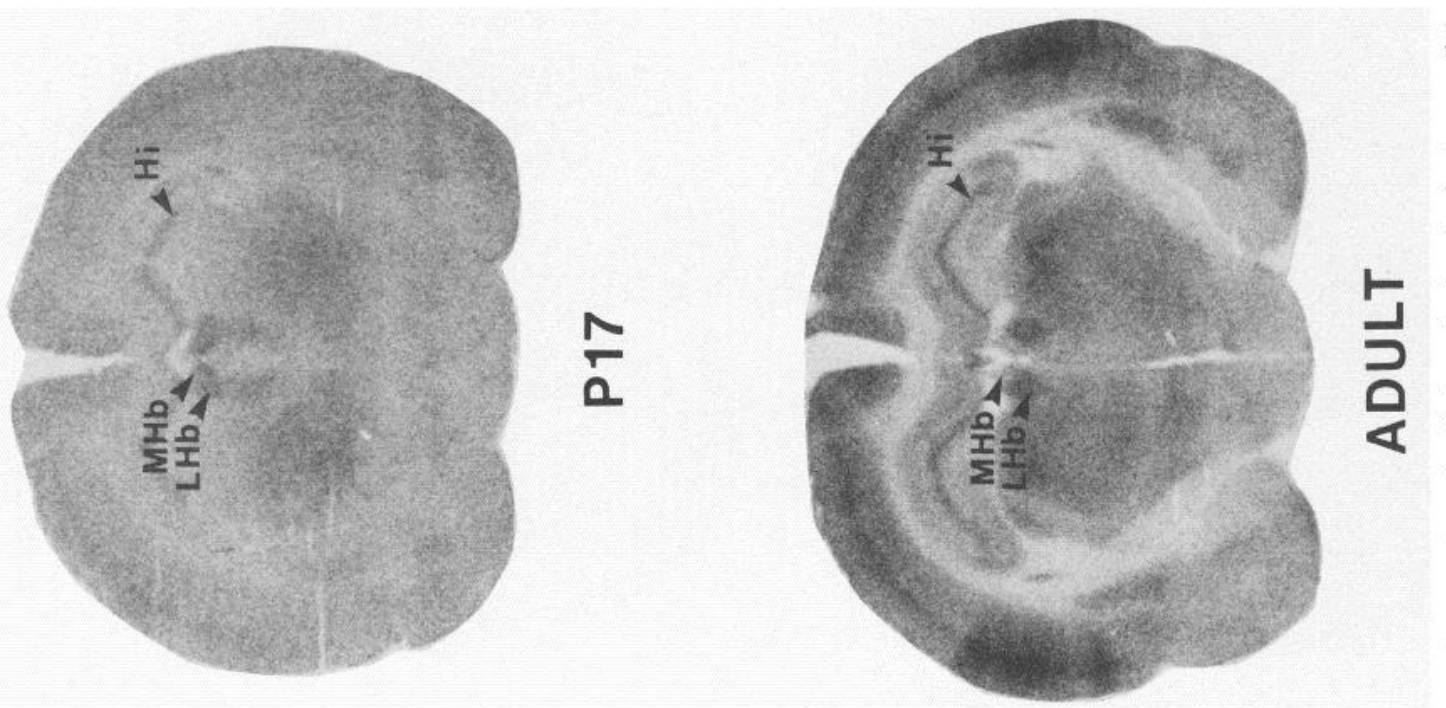

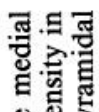

ฏ氙

.

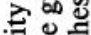

突

-

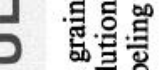

?

낭

表䓃

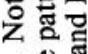

ํㅗㄹ

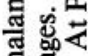

जั

‡氖

형
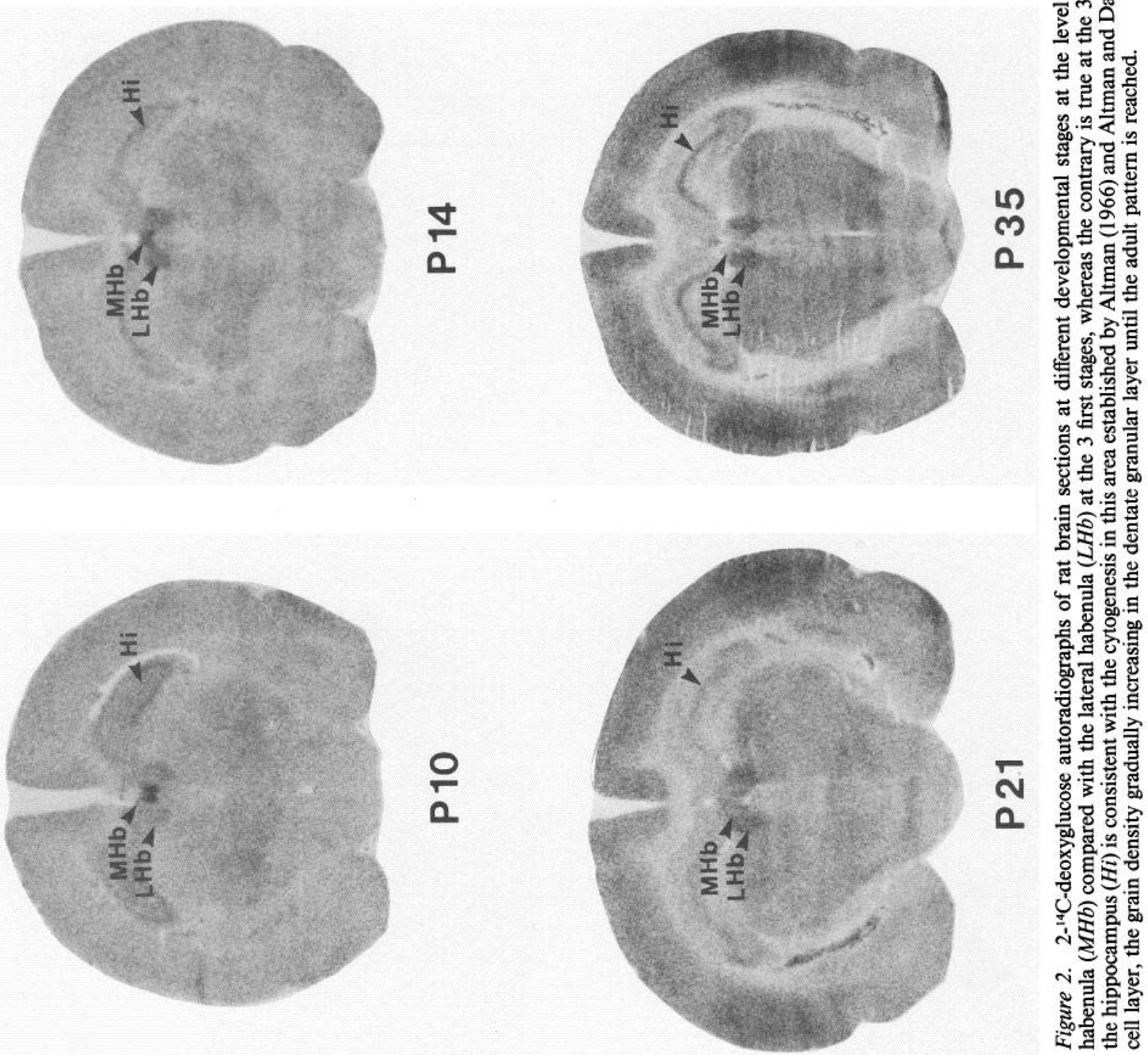

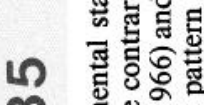

(

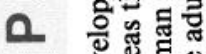
氙焉

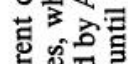
悹嵒 的急 诌密 용 进 的电 

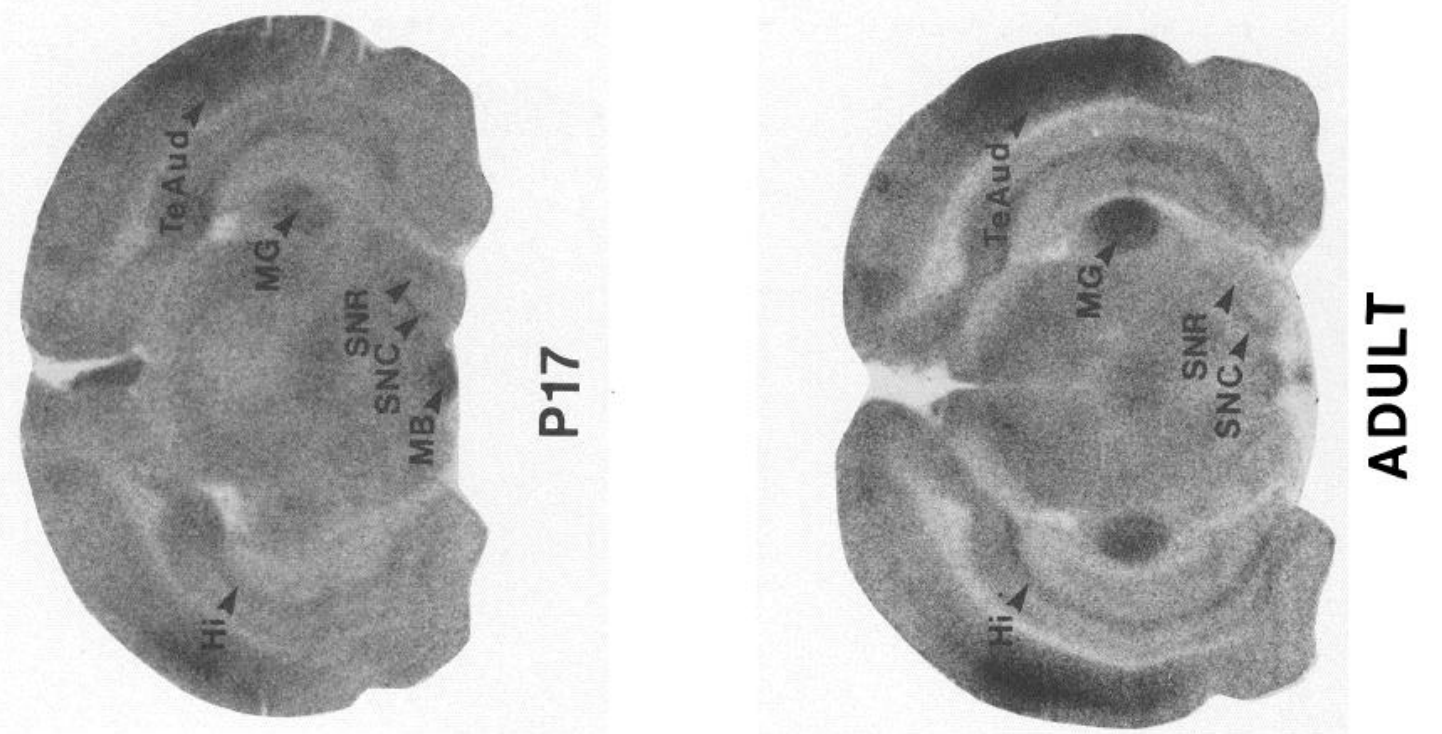

열 영

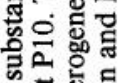

的新

o은

$\vdash \quad$

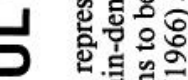

.

政

경멸

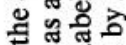

论

2 을

กั

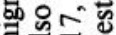

त㓉

政宛

政

列.
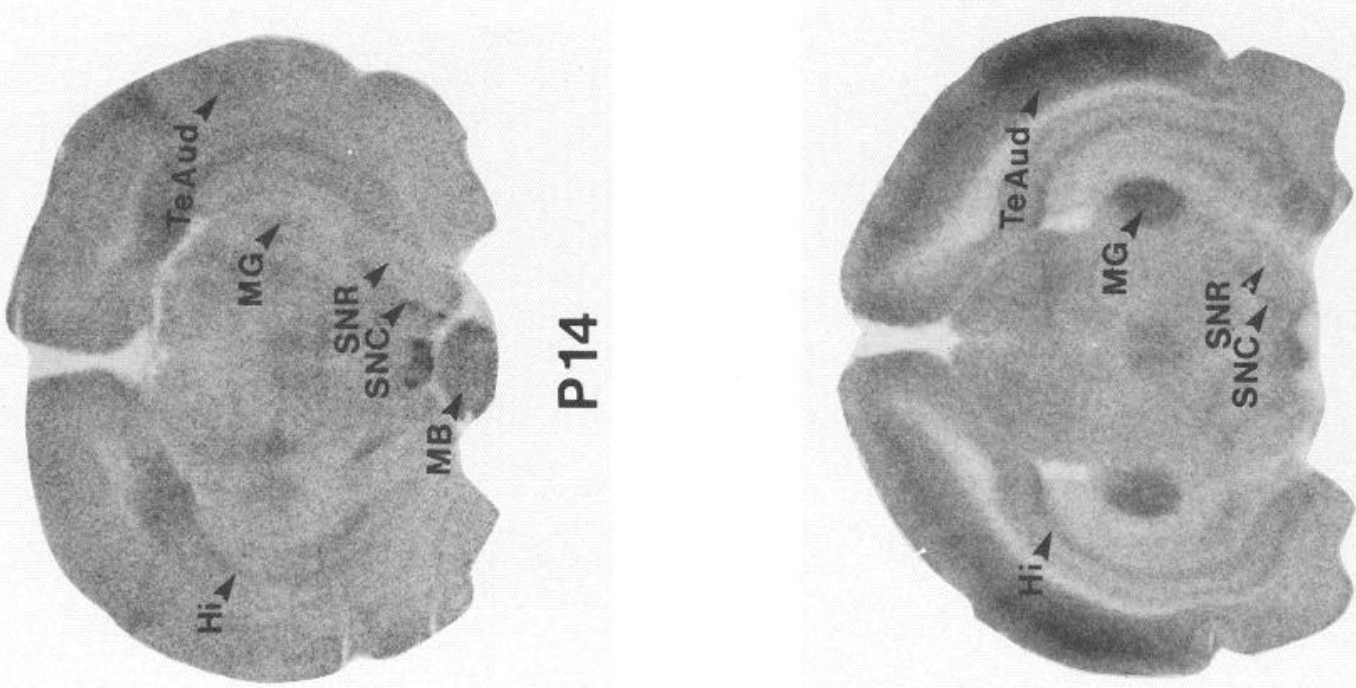

ts.

of

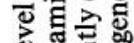

음

옹

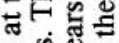

这宓

10 )

$\infty$ s.

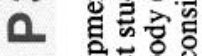

옹

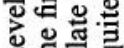

홍

猔.

공

항

ธั

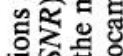

论

क

뎔

(5)

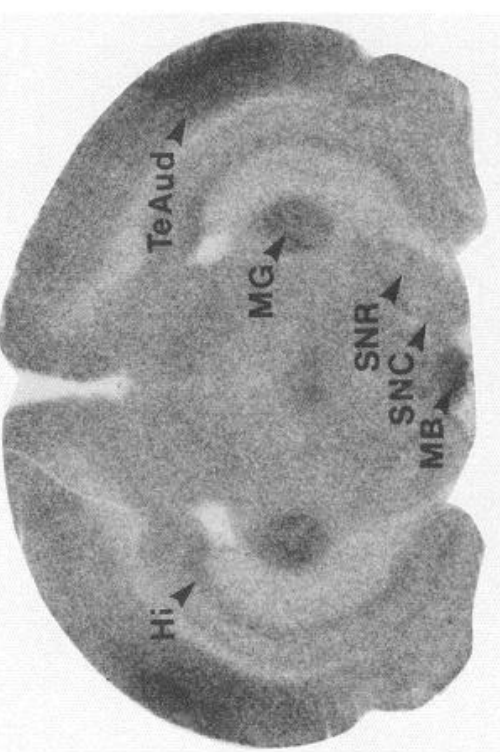

5

웡

.

응

चु

응

글.

\%

స

和近

Q 10 용

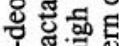

نํ.

त 8

的些

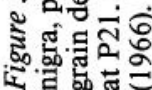



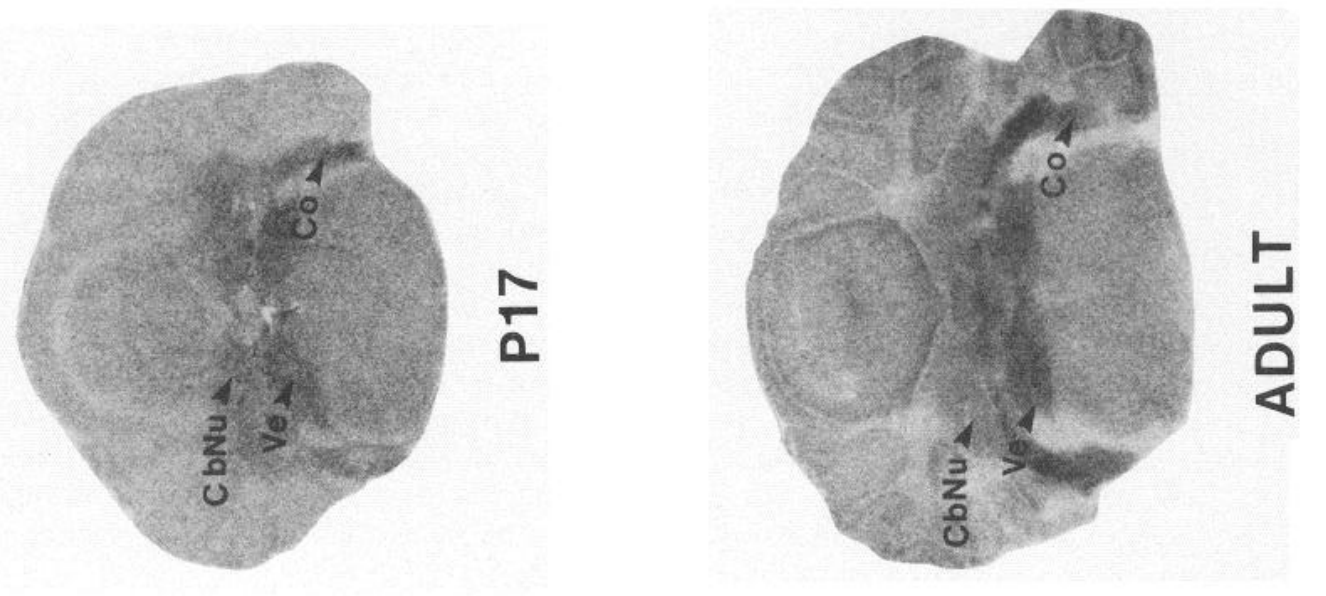

‡

I

产

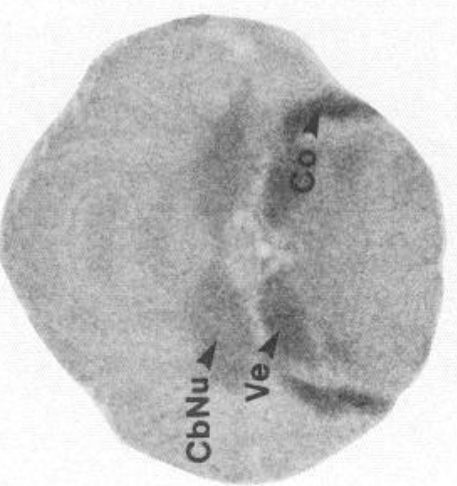

$\frac{1}{2}$

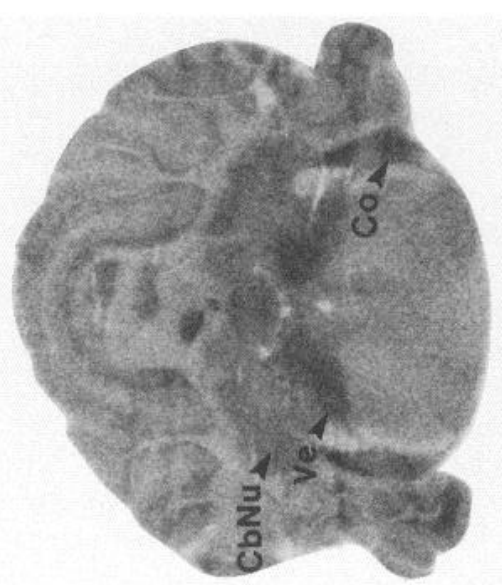

ก

ก

ค.

鄀

兽兘

흘

产产

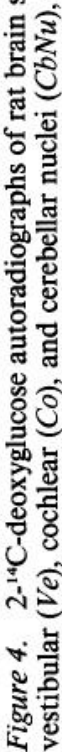

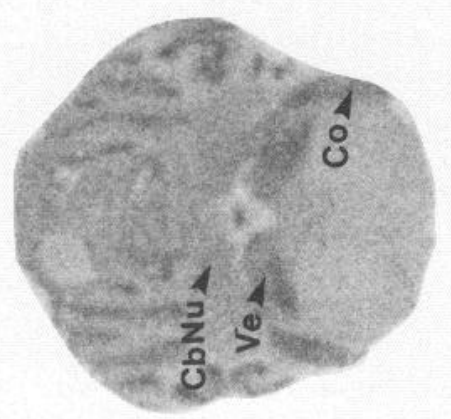

$\frac{1}{2}$

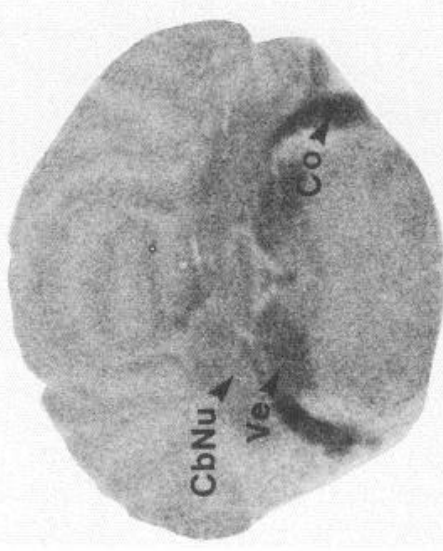

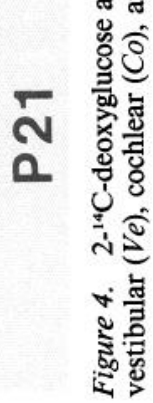


essentially differentiated and heterogeneous around weaning time, i.e., $21 \mathrm{~d}$ after birth.

The low and homogeneous LCMRglu in the brain of the 10$\mathrm{d}$-old rat, as determined by the $2-{ }^{14} \mathrm{C}$-deoxyglucose technique, confirms the low rate of glucose utilization measured in vitro in the immature rat brain as a whole (Himwich, 1951). This low rate can be related to the relative immaturity of the brain at this stage. Indeed, at $10 \mathrm{~d}$ after birth, the general cellular immaturity in all areas and strata of the brain is still pronounced, and there is no marked degree of neocortical influence upon behavior (Tilney, 1933). In contrast, LCMRglu in most other species studied appears to be more differentiated in newborns than in 10-d-old rats. This difference can be related to the variability in the degree of maturity of different species at birth. LCMRglu appears to be distributed in a narrower range in the brain of the newborn dog (Duffy et al., 1982) and monkey (Kato et al., 1980; Kennedy et al., 1982) than in sheep (Abrams et al., 1984), which is a more precocious species than the other two. In human infants, LCMRglu at birth also appears to be more differentiated than in the rat (Chugani and Phelps, 1986; Chugani et al., 1987); the newborn rat is indeed more immature, and a human newborn corresponds approximately to a 7 - to 8-d-old rat (Dobbing, 1974; Alling, 1985).

\section{Auditory system}

No reactions to sound can be elicited at $10 \mathrm{~d}$. Between 10 and $14 \mathrm{~d}$, glucose utilization increases in 4 auditory areas (Table 2) at a period corresponding to the maturation of auditory function. Indeed, the opening of the external auditory meatus normally occurs 12-13 d after birth in the rat, at which time there is a marked increase in sensitivity to a wide range of tones (Crowley and Hepp-Reymond, 1966; Rose and Ellingson, 1970). This anatomical and functional maturation is paralleled by the appearance of electrical responses to auditory stimuli at about 12-14 d of age (Mourek et al., 1967; Mysliveček, 1970). By P14, the total sensitivity to sound has reached its adult level (Crowley and Hepp-Reymond, 1966). The further maturation of auditory function is reflected by the increased LCMRglu at the highest integrating level, the auditory cortex, at P17.

\section{Visual system}

In the rat, the eyelids usually open at about P15 (Tilney, 1933), the stage at which the electroretinogram shows a characteristic pattern (Dowling and Sidman, 1962; Rose and Ellingson, 1970). Visual cvoked potentials can be clicited at $14 \mathrm{~d}$, and after $18 \mathrm{~d}$, photically evoked discharges are apparent (Klingberg and Schwartze, 1966; Mourek et al., 1967; Mysliveček, 1970). The acquisition of visual function translates into an increase in LCMRglu in one visual area, the lateral geniculate body between $\mathrm{P} 14$ and $\mathrm{P} 17$.

\section{Correlation between LCMRglu and behavior in suckling rat}

By $17 \mathrm{~d}$ after birth, significant developments in the animal's somatic behavior are apparent. The capacity of rats to explore their environment, as well as the variety of their apparently playful activities, is indicative of a more highly organized behavior, which implies the functional participation of well-differentiated neural mechanisms in the neocortex, especially in the parietal area (Tilney, 1933). These behavior changes translate into an increase in LCMRglu between P14 and P17 in the frontoparietal cortex, both the somatosensory and motor areas. These changes are paralleled by increases of LCMRglu in 3 motor areas, including the ventroanterior nucleus of the thalamus (Table 5). LCMRglu is also increased at $17 \mathrm{~d}$ in a limbic part of the thalamus, the mediodorsal nucleus (Table 3). These early increases in thalamic LCMRglu are comparable to the high levels of LCMRglu measured in the thalamus of human infants (Chugani and Phclps, 1986; Chugani et al., 1987).

Most of the characteristics of the adult rat are apparent in the behavior of the 20-d-old rat. All parts of the neocortex have by this time attained a degree of differentiation sufficient to make them available for the reception of somesthetic, auditory, and visual impulses. In addition, by day 20 , the most prominent change in the cerebral hemispheres is a general size increase in the neocortex (Tilney, 1933). This high degree of development translates into a $50 \%$ increase in the average rate of cerebral glucose utilization and into a general big increase in LCMRglu in almost all studied structures. By P21, the average rate of glucose utilization reaches $76 \%$ of the adult value, and LCMRglu begins to be differentiated and quite heterogeneous throughout the whole brain.

\section{Correlation between LCMRglu and electrical activity during rat postnatal development}

All the anatomical, behavioral, and functional changes occurring during the first 3 postnatal weeks are well correlated to the development of brain electrical activity. The electroencephalogram, which first appears at $6 \mathrm{~d}$, is slow and low in amplitude. It speeds up and increases in voltage near the end of the second postnatal week and takes on the features of adult spectral composition at the end of the third postnatal week (Tuge et al., 1960; Deza and Eidelberg, 1967), when LCMRglu is high throughout the rat brain. Also, increases in amplitude of steady potentials and membrane potentials are found between the P10 and P20 postnatal day in the cerebral cortex, striatum, and hippocampus but at P20 these potentials have still not reached adult levels (Bureš, 1957; Mareš, 1964).

\section{Correlation between LCMRglu and biochemical maturation of the rat brain}

The period from birth to $\mathrm{P} 24$ is considered to be a critical period in brain development in the rat. All the morphological, anatomical, and biochemical changes occurring during this period are paralleled by functional changes.

The advanced state of maturation of the rat brain at weaning time, i.e., $21 \mathrm{~d}$, is reflected by high LCMRglu in all cerebral structures. This age corresponds to the end of the active brain growth spurt and to the peak of myelination (Davison and Gregson, 1962, 1966; Norton and Poduslo, 1973). Also, at P21, the DNA content of the rat brain reaches its adult level (Fisch and Winick, 1969) and $90 \%$ of the adult value of gangliosides has been attained by P24 (Vanier et al., 1971). The marked increase in LCMRglu taking place between P10 and P21 in the present study confirms previous data on the maturation of glucose carbon utilization by the brain as a whole over the same developmental period (Gaitonde and Richter, 1966; Cocks et al., 1970). The conversion of carbon glucose into amino acids is not very active in newborn rats and in animals less than $12 \mathrm{~d}$ old; it progressively increases after P12, and by 19-22 d after birth, the rate of conversion of glucose carbon into amino acids is similar to that in adults (Gaitonde and Kichter, 1966; Cocks et al., 1970). Over the same maturation period, the flux through the tricarboxylic acid cycle is morc than doubled (Balázs, 1971) and the activities of the enzymes of the glycolytic pathway and 
the tricarboxylic acid cycle are largely increased and their developmental curves are similar to those for the conversion of glucose carbon into amino acids (Hamburgh and Flexner, 1957; Garcia-Argiz et al., 1967; Gregson and Williams, 1969; Swaiman et al., 1970; Schwark et al., 1972). In addition, the rate of conversion of glucose carbon into amino acids, the activity of oxidative mitochondrial enzymes, and the oxygen consumption of the developing rat brain (Fazekas et al., 1951) all undergo sigmoid increases that parallel those noted for the postnatal changes in LCMRglu in the developing rat.

Glucose has been shown to be the predominant, nearly exclusive energy substrate in the brain of normal adult animals. On the contrary, the brain of immature animals is able to use a wide range of substrates. During the suckling period in the rat, ketone bodies are widely used by the brain and the cerebral arteriovenous differences of $\beta$-hydroxybutyrate and acetoacetate are 3-4 times higher in the 16- to 22-d-old rat than in the adult one (Hawkins et al., 1971). This important uptake and utilization of ketone bodies by the brain of young animals is directly related to the high activity of ketone body metabolizing enzymes in the immature rat brain (Klee and Sokoloff, 1967; Krebs et al., 1971; Page et al., 1971). From the estimated rates of oxidative metabolism of both glucose and ketone bodies, it appears that ketone bodies can account for at least $30 \%$ of the total in suckling rats (Cremer and Heath, 1974; Dahlquist and Persson, 1976; Cremer, 1982). These ketone bodies are more efficient precursors than glucose for the biosynthesis of cerebral amino acids in the brain of immature animals (DeVivo et al., 1973, 1975). They are also very active precursors of lipid biosynthesis in the brain of suckling rats (Patel and Owen, 1977; Yeh et al., 1977) and play an important role in the processes of myelination and development of the immature brain.

\section{Correlation between LCMRglu and the evolution of behavior and brain functional activity of the rat after weaning}

At weaning time, however, even if some brain metabolic processes and biochemical constituents have reached a level close to that of the adult, the rat brain is still undergoing quite active maturation. For example, the cerebral cortex has not attained its full structural specialization by P21. The end of the most rapid phase of myelination takes place around P30 (Norton and Poduslo, 1973), and the behavior of the rat is still changing during this period. The playful activities of the rats are complete by the stage of $35 \mathrm{~d}$, and sexual maturity is attained by $50-60$ d (Tilney, 1933). Adult patterns of cortical electrical activity are only reached by 8 weeks (Yoshii and Tsukiyama, 1951; Deza and Eidelberg, 1967). This maturation phase still translates into a $25 \%$ increase in the average rate of glucose utilization by the brain as a whole between P21 and P35. The increases in LCMRglu are widely distributed throughout the brain, affecting, for example, all cortical areas and most hypothalamic and motor structures. Between P35 and adulthood, the average rate of cerebral glucose utilization remains unchanged, but increases in LCMRglu do occur in scattered areas. For example, LCMRglu continues to increase in some cortical areas, whereas no further changes are noted in hypothalamic structures.

\section{Interspecies comparison of postnatal development of cerebral functional activity}

In contrast to the sigmoid rise in LCMRglu observed in the developing rat, rates of cerebral functional activity in children (Kennedy and Sokoloff, 1957; Chugani et al., 1987) and in im- mature dogs (Kennedy et al., 1972) exceed adult values. These transiently high rates of functional activity may reflect the summation of energy demands for energy metabolism together with biosynthetic processes associated with growth and matuation. The postnatal changes in the rates of cerebral blood flow in the dog confirm the peaks of cerebral oxygen consumption in the dog found in vitro in early postnatal life (Himwich and Fazekas, 1941).

However, in the rat brain, oxygen consumption (Fazekas et al., 1951), oxidative mitochondrial enzyme activity (Hamburgh and Flexner, 1957; Garcia-Argiz et al., 1967; Gregson and Williams, 1969; Swaiman et al., 1970; Schwark et al., 1972), the rate of conversion of glucose carbon into amino acids (Gaitonde and Richter, 1966; Cocks et al., 1970), and LCMRglu undergo a progressive sigmoid rise in all structures until mature levels of activity are reached.

This interspecies difference may be related to the rate of utilization of ketone bodies by the immature brain. Indeed, in the developing rat, the circulating concentration of ketone bodies is high, and these substrates are widely used by the brain (Hawkins et al., 1971; DeVivo et al., 1973, 1975; Pereira de Vasconcelos et al., 1987). The brain of human newborns and infants is also able to take up and oxidize ketone bodies (Kraus et al., 1974; Dahlquist et al., 1976), but between 3 and 9 years of age, when the LCMRglu in children reaches its highest levels (Chugani et al., 1987), glucose has already become the predominant substrate for brain metabolism. Thus, the difference in postnatal changes in LCMRglu during development in children and rat pups may be correlated with the fact that at the time of the active brain spurt and intense myelination, i.e., mainly until weaning time in the rat and throughout the first decade in the child (Yakovlev and Lecours, 1967; Holland et al., 1986), the rat brain is still dependent upon ketone bodies, which are better precursors than glucose for lipid biosynthesis (Patel and Owen, 1977; Yeh et al., 1977); however, the brain of a 3 to 9 year old child depends entirely on glucose for its energy metabolism and all its biosynthesis.

\section{LCMRglu in the medial habenula}

In contrast to all other brain structures considered in the present study, no change in LCMRglu over the developmental period studied was noted in 2 limbic structures, the medial habenula (Table 4, Fig. 2) and the habenulointerpeduncular tract or fasciculus retroflexus (data not shown), which both appear as black areas between 10 and $17 \mathrm{~d}$. LCMRglu in these 2 areas raises the question of the role played by this limbic circuitry in rat pups. They may be correlated to the quite high rates of glucose utilization noted in the raphe nuclei as early as P10 (Table 3) since this structure has been shown to project to the habenula (Törk, 1985). In addition, the relatively high LCMRglu at this stage in the monoamine and serotonin cell groupings (i.e., substantia nigra pars compacta, raphe nuclei, and locus coeruleus), as compared with the other brain areas, may be a reflection of the quite advanced maturity of monoamine and serotonin neurons at this stage of development (Lauder and Bloom, 1975; Lanier et al., 1976).

\section{Conclusion}

As previously shown in several studies performed with autoradiographic techniques (Kennedy et al., 1972, 1982; Abrams et al., 1984; Chugani et al., 1987), a rise in metabolic rate of a particular structure generally marks the time at which it begins 
to contribute to the animal's behavior. This could also be demonstrated in the developing rat, and the results of the present study show that there is a good correlation between increases in local rates of glucose utilization and the behavioral, anatomical, and functional changes known to occur in the brain of the rat during postnatal development. In addition, in the rat, LCMRglu undergoes a sigmoid rise during postnatal development, whereas in other species there is a peak in functional activity in the immature brain before the adult level is reached (Kennedy and Sokoloff, 1957; Kennedy et al., 1972; Chugani et al., 1987). This interspecies difference may originate in the active utilization of ketone bodies by the developing rat brain at the period of active brain growth spurt and intense myelination.

\section{References}

Abrams, R. M., M. Ito, J. E. Frisinger, C. S. Patlak, K. D. Pettigrew, and C. Kennedy (1984) Local cerebral glucose utilization in fetal and neonatal sheep. Am. J. Physiol. 246: R608-R618.

Alling, C. (1985) Biochemical maturation and the concept of vulnerable periods. In Alcohol and the Developing Brain, U. Rydberg et al., eds., pp. 5-10, Raven, New York.

Altman, J. (1966) Autoradiographic and histological studies of postnatal neurogenesis. II. A longitudinal investigation of the kinetics, migration and transformation of cells incorporating tritiated thymidine in infant rats, with special reference to postnatal neurogenesis in some brain regions. J. Comp. Neurol. 128: 431-474.

Altman, J., and G. D. Das (1966) Autoradiographic and histological studies of postnatal neurogenesis. I. A longitudinal investigation of the kinetics, migration and transformation of cells incorporating tritiated thymidine in neonate rats, with special reference to postnatal neurogenesis in some brain regions. J. Comp. Neurol. 126: 337-390.

Balázs, R. (1971) Biochemical effects of thyroid hormones in the developing rat. In Cellular Aspects of Neural Growth and Differentiation (UCLA Forum in Medical Sciences 14), D. C. Pease, ed., pp. 273311, University of California Press, Los Angeles.

Bureš, J. (1957) The ontogenetic development of steady potential differences in the cerebral cortex in animals. Electroencephalogr. Clin. Neurophysiol. 7: 85-98.

Chugani, H. T., and M. E. Phelps (1986) Maturational changes in cerebral function in infants determined by ${ }^{18} \mathrm{FDG}$ positron emission tomography. Science 231: 840-843.

Chugani, H. T., M. E. Phelps, and J. C. Mazziotta (1987) Positron emission tomography study of human brain functional development Ann. Neurol. 22: 487-497.

Cocks, J. A., R. Balázs, A. L. Johnson, and J. T. Eayrs (1970) Effect of thyroid hormone on the biochemical maturation of rat brain: Conversion of glucose-carbon into amino acids. J. Neurochem. 17: 12751285.

Cremer, J. E. (1982) Review: Substrate utilization and brain development. J. Cereb. Blood Flow Metabol. 2: 394-407.

Cremer, J. E., and D. F. Heath (1974) The estimation of rates of utilization of glucose and ketone bodies in the brain of the suckling rat using compartmental analysis of isotopic data. Biochem. J. 142: $527-544$

Crowley, D. E., and M. C. Hepp-Reymond (1966) Development of cochlear function in the ear of the infant rat. J. Comp. Physiol. Psychol. 62: 427-432.

Dahlquist, G., and B. Persson (1976) The rate of cerebral utilization of glucose, ketone bodies and oxygen. A comparative in vivo study in infant and adult rats. Pediatr. Res. 10: 910-917.

Davison, A. N., and N. A. Gregson (1962) The physiological role of cerebron sulphuric acid (sulfalide) in the brain. Biochem. J. 85: 558568.

Davison, A. N., and N. A. Gregson (1966) Metabolism of sulfolipids in the rat brain. Biochem. J. 98: 915-922.

DeVivo, D. C., M. P. Leckie, and H. C. Agrawal (1973) The differential incorporation of $\beta$-hydroxybutyrate and glucose into brain glutamate in the newborn rat. Brain Res. 55: 485-490.

DeVivo, D. C., M. P. Leckie, and H. C. Agrawal (1975) D- $\beta$-hydroxybutyrate: A major precursor of amino acids in developing rat brain. J. Neurochem. 25: 161-170.
Deza, L., and E. Eidelberg (1967) Development of cortical electrical activity in the rat. Exp. Neurol. 17: 425-438.

Dobbing, J. (1974) The later growth of the brain and its vulnerability. Pediatrics 53: 2-6.

Dowling, J. E., and R. L. Sidman (1962) Inherited retinal dystrophy in the rat. J. Cell Biol. 14: 73-110.

Duffy, T. E., S. J. Kohlc, and R. C. Vannucci (1975) Carbohydrate and energy metabolism in perinatal rat brain: Relation to survival to anoxia. J. Neurochem. 24: 271-276.

Duffy, T. E., M. Cavazzuti, N. F. Cruz, and L. Sokoloff (1982) Local cerebral glucose metabolism in newborn dogs: Effects of hypoxia and halothane anesthesia. Ann. Neurol. 11: 233-246.

Fazekas, J. F., F. A. D. Alexander, and H. E. Himwich (1941) Tolerance of the newborn to anoxia. Am. J. Physiol. 134: 281-287.

Fazekas J. F., F. B. Graves, and R. W. Alman (1951) Influence of thyroid on cerebral metabolism. Endocrinology 48: 169-174.

Fisch, I., and M. Winick (1969) Effect of malnutrition on regional growth of the developing rat brain. Exp. Neurol. 25: 534-540.

Gaitonde, M. K., and D. Richter (1966) Changes with age in the utilization of glucose carbon in liver and brain. J. Neurochem. 13: 1309-1318.

Garcia-Argiz, C. A., J. M. Pasquini, B. Kaplun, and C. Gomez (1967) Hormonal regulation of brain development. II. Effect of neonatal thyroidectomy on succinate dehydrogenase and other enzymes in the developing cerebral cortex and cerebellum of the rat. Brain Res. 6 : 635-646.

Garfunkel, J. M., H. W. Baird, and J. Ziegler (1954) The relationship of oxygen consumption to cerebral functional activity. J. Pediatr. 44: 64-72.

Gregoire, N. M., A. Gjedde, F. Plum, and T. E. Duffy (1978) Cerebral blood flow and cerebral metabolic rates for oxygen, glucose, and ketone bodies in newborn dogs. J. Neurochem. 30: 63-69.

Gregoire, N., R. Pontier, and G. Salamon (1981) Local cerebral glucose utilization in the newborn brain. Eur. Neurol. 20:162-168.

Gregson, N. A., and P. L. Williams (1969) A comparative study of brain and liver mitochondria from new-born and adult rats. J. Neurochem. 16: 617-626.

Hamburgh, M., and L. B. Flexner (1957) Physiological differentiation during morphogenesis. XXI. Biochemical and physiological differentiation during morphogenesis. Effect of hypothyroidism and hormone therapy on enzyme activities of the developing cerebral cortex of the rat. J. Neurochem. 1:279-288.

Hawkins, R. A., D. H. Williamson, and H. A. Krebs (1971) Ketonebody utilization by adult and suckling rat brain in vivo. Biochem. J. 122: 13-18.

Hernandez, M. J., R. W. Brennan, R. C. Vannucci, and G. S. Bowman (1978) Cerebral blood flow and oxygen consumption in the newborn dog. Am. J. Physiol. 234: R209-R215.

Himwich, H. E. (1951) Brain Metabolism and Cerebral Disorders, pp. 157-163, Williams \& Wilkins, Baltimore.

Himwich, H. E., and J. F. Fazekas (1941) Comparative studies of the metabolism of the brain of infant and adult dogs. Am. J. Physiol. 132: 454-459.

Holland, B. A., D. K. Haas, D. Norman, M. Brant-Zawadzki, and T. H. Newton (1986) MRI of normal brain maturation. Am. J. Neuroradiol. 7: 201-208.

Kato, M., B. L. Malamut, D. F. Caveness, S. Hosokawa, S. Wakisawa, and R. R. O'Neill (1980) Local cerebral glucose utilization in newborn and pubescent monkeys during focal motor seizures. Ann. Neurol. 7: 204-212.

Kennedy, C., and L. Sokoloff (1957) An adaptation of the nitrous oxide method to the study of the cerebral circulation in children; normal values for cercbral flood flow and cerebral metabolic rate in childhood. J. Clin. Invest. 36: 1130-1137.

Kennedy, C., J. D. Grave, J. W. Jehle, and L. Sokoloff (1972) Changes in blood flow in the component structures of the dog brain during postnatal maturation. J. Neurochem. 19: 2423-2433.

Kennedy, C., O. Sakurada, M. Shinohara, J. Jehle, and L. Sokoloff (1978) Local cerebral glucose utilization in the normal conscious macaque monkey. Ann. Neurol. 4: 293-301.

Kennedy, C., O. Sakurada, M. Shinohara, and M. Miyanka (1982) Local cerebral glucose utilization in the newborn macaque monkey. Ann. Neurol. 12: 333-340.

Kirk, R. E. (1968) Experimental Design: Procedures for the Behavioral Sciences, Brooks/Cole, Belmont, CA. 
Klee, C. B., and L. Sokoloff (1967) Changes in D(-)- $\beta$-hydroxybutyric dehydrogenase activity during brain maturation in the rat. $\mathrm{J}$. Biol. Chem. 242: 3880-3883.

Klingberg, F., and P. Schwartze (1966) Uber photisch ausgeloste Nachentladungen im visuellen Cortex der Ratte während der Ontogenese. Pflueger's Arch. Ges. Physiol. 292: 90-99.

Kraus, H., S. Schlcnker, and D. Schwedesky (1974) Developmental changes of cerebral ketone body utilization in human infants. Hoppe Seyler's Z. Physiol. Chem. 355: 164-170.

Krebs, H. A., D. H. Williamson, M. W. Bates, M. A. Page, and R. A. Hawkins (1971) The role of ketone bodies in caloric homeostasis. Adv. Enzyme Regul. 9: 387-409.

Lanier, L. P., A. J. Dunn, and C. Van Hartesveldt (1976) Development of neurotransmitters and their function in brain. In Reviews of Neuroscience, Vol. 2, S. Ehrenpreis and I. I. Kopin, eds., pp. 195-256 Raven, New York.

Lauder, J. M., and F. E. Bloom (1975) Ontogeny of monoamine neurons in the locus coeruleus, raphe nuclei and substantia nigra of the rat. II Synaptogenesis. J. Comp. Neurol. 163: 251-264.

Mareš, P. (1964) Ontogenetic development of membrane potentials in tclenccphalic structures in the rat. Physiol. Bohemoslov. 13: 256262.

Mellanby, J., and D. H. Williamson (1974) Acetoacetate. In Methods of Enzymatic Analysis, Vol. 4, H. U. Bergmeyer and K. Gawehn, eds., pp. 1840-1843, Academic, New York.

Mourek, J., W. A. Himwich, J. Mysliveček, and D. Callison (1967) The role of nutrition in the development of evoked cortical responses in the rat. Brain Res. 6: 241-251.

Mysliveček, J. (1970) Electrophysiology of the developing brain. Central and eastern European contributions. In Developmental Neurobiology, W. A. Himwich, ed., pp. 475-527, Charles C. Thomas, Springfield, IL.

Norton, W. T., and S. E. Poduslo (1973) Myelin in rat brain: Changes in myelin composition during brain maturation. J. Neurochem. 21 . $759-773$.

Page, M. A., H. A. Krebs, and D. H. Williamson (1971) Activities of enzymes of ketone-body utilization in brain and other tissues of suckling rats. Biochem. J. 121: 49-53.

Patel, M. S., and O. E. Owen (1977) Development and regulation of lipid synthesis from ketone bodies by rat brain. J. Neurochem. 28 : 109-114.

Paxinos, G., and C. Watson (1982) The Rat Brain in Stereotoxic Coordinates, Academic, New York.

Pereira de Vasconcelos, A., H. Schroeder, and A. Nehlig (1987) Effects of early chronic phenobarbital treatment on the maturation of energy metabolism in the developing rat brain II. Incorporation of $\beta$-hydroxybutyrate into amino acids. Dev. Brain Res. 36: 231-236.

Rose, G. H., and R. J. Ellingson (1970) Ontogenesis of evoked potentials. In Developmental Neurobiology, W. A. Himwich, ed., pp. 393-440, Charles C. Thomas, Springfield, IL.

Schwark, W.S., R. L. Singhal, and G. M. Ling (1972) Metabolic control mechanisms in mammalian systems. Regulation of key glycolytic enzymes in developing brain during experimental cretinism. J. Neurochem. 19: 1171-1182.

Sherwood, N. M., and P. S. Timiras (1970) A Stereotaxic Atlas of the Developing Rat Brain, University of California Press, Berkeley.

Sokoloff, L. (1981) Localization of functional activity in the central nervous system by measurement of glucose utilization with radioactive deoxyglucose. J. Cereb. Blood Flow Metabol. 1: 7-36.

Sokoloff, L., M. Reivich, C. Kennedy, M. H. DesRosiers, C. S. Patlak, K. D. Pettigrew, O. Sakurada, and M. Shinohara (1977) The $\left[{ }^{14} \mathrm{C}\right]-$ deoxyglucose method for the measurement of local cerebral glucose utilization: Theory, procedure, and normal values in the conscious and anesthetized albino rat. J. Neurochem. 28: 897-916.

Swaiman, K. F., J. M. Daleiden, and R. N. Wolfe (1970) The effect of food deprivation on enzyme activity in developing brain. J. Neurochem. 17: 1387-1391.

Tilney, F. (1933) Behavior in its relation to the development of the brain. II. Correlation between the development of the brain and behavior in the albino rat from embryonic states to maturity. Bull. Neurol. Inst. N.V. 2: 252-358.

Törk, I. (1985) Raphe nuclei and serotonin containing system. In The Rat Nervous System, Vol 2, Hindbrain and Spinal Cord, G. Paxinos, ed., pp. 43-78, Academic, Sydney, Australia.

Tuge, H., Y. Kanayama, and H. Y. Chang (1960) Comparative studies on the development of the EEG. Jpn. J. Physiol. 10: 211-220.

Vanier, M. T., M. Holm, R. Ohman, and L. Svennerholm (1971) Developmental profiles of gangliosides in human and rat brain. J. Neurochem. 18: 581-592.

Williamson, D. H., and J. Mellanby (1974) D-(-)-3-hydroxybutyrate. In Methods of Enzymatic Analysis, Vol. 4, H. U. Bergmeyer and K. Gawehn, eds., pp. 1836-1839, Academic, New York.

Yakovlev, P. I., and A. R. Lecours (1967) The myelogenetic cycles of regional maturation of the brain. In Regional Development of the Brain in Early Life, A. Minkowski, ed., pp. 3-70, Davis, Philadelphia.

Yeh, Y. Y., V. L. Streuli, and P. Zee (1977) Ketone bodies serve as important precursors of brain lipids in the developing rat. Lipids 12 : 957-964.

Yoshii, N., and K. Tsukiyama (1951) Normal EEG and its development in the white rat. J. Jpn. Physiol. 2: 34-38. 